\title{
Interplanetary coronal mass ejections in the near-Earth solar wind during the minimum periods following solar cycles 22 and 23
}

\author{
E. K. J. Kilpua ${ }^{1}$, C. O. Lee ${ }^{2}$, J. G. Luhmann ${ }^{2}$, and Y. Li $^{2}$ \\ ${ }^{1}$ Department of Physics, Division of Geophysics and Astronomy, University of Helsinki, Finland \\ ${ }^{2}$ Space Sciences Laboratory, University of California, Berkeley, CA, USA
}

Received: 20 November 2010 - Revised: 10 June 2011 - Accepted: 5 August 2011 - Published: 30 August 2011

\begin{abstract}
In this paper we examine the occurrence rates and properties of interplanetary coronal mass ejections (ICMEs) and solar activity levels during the minima following solar cycle 22 (January 1995-December 1997) and 23 (January 2007-April 2010) minima using observations from the OMNI data base. Throughout the minimum following cycle 22 the CME and ICME rates roughly tracked each other, while for the minimum following cycle 23 they diverged. During the minimum after solar cycle 23, there were large variations in the streamer belt structure. During the lowest activity period of cycle 23 (based on sunspot numbers), the ICME rate was about four times higher than during a similar activity period of cycle 22 . We propose that this relatively high ICME rate may be due to CME source regions occurring at lower heliolatitudes and due to equatoward deflection of slow and weak CMEs originating from the midand high-heliolatitudes. The maximum magnetic fields of the ICMEs identified during the minimum following cycle 23 were $\sim 30 \%$ lower and their radial widths were $\sim 15 \%$ lower compared to the ICMEs observed during the minimum following solar cycle 22. The weak and small ICMEs may result from intrinsically weak CMEs and/or they may represent stronger CMEs that are encountered far away from the center.
\end{abstract}

Keywords. Interplanetary physics (Interplanetary magnetic fields)

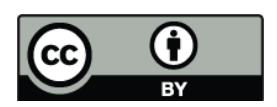

Correspondence to: E. K. J. Kilpua (emilia.kilpua@helsinki.fi)

\section{Introduction}

Coronal mass ejections (CMEs) are magnetized plasma clouds that are ejected from the Sun and propagate out into the heliosphere. When CMEs are observed in-situ in the interplanetary medium they are referred to as interplanetary CMEs (ICMEs).

The number of CMEs varies with the solar activity cycle, increasing by an order of magnitude from minimum to maximum (St. Cyr et al., 2000; Gopalswamy, 2006). While coronagraphs record CMEs launched to all heliospheric latitudes, the estimates of the ICME rate are typically based on single-spacecraft measurements carried out close to the ecliptic plane. The ICME rate increases from one event every three months at solar minimum to about one event per week during solar maximum (Richardson and Cane, 2010). The near-ecliptic ICME rate generally correlates with the CME rate, but deviations have been reported. Riley et al. (2006) showed that the CME rate and the near-ecliptic ICME rate tracked each other during the minimum after solar cycle 22 (hereafter, SC 22 minimum), but diverged during the ascending phase of solar cycle 23 . The work by Kilpua et al. (2009b) using multi-spacecraft observations from the Solar Terrestrial Relation Observatory (STEREO) (Kaiser et al., 2007) and the Wind and ACE spacecraft located the Lagrangian point L1 showed that the ICME and CME rates diverged during 2007-2008, coinciding with the minimum after solar cycle 23 (hereafter, SC 23 minimum).

The divergence of the CME and near-ecliptic ICME rates can be explained by the solar cycle variations in the CME source locations at the Sun. During solar minimum the CME source locations are clustered close to the solar equator while during the solar cycle ascending phase the source locations are located at higher latitudes (Hundhausen et al., 1993; St. Cyr et al., 2000). There are several effects that can contribute

Published by Copernicus Publications on behalf of the European Geosciences Union. 
to the variations in the source latitudes of CMEs. The largescale solar magnetic structures from where CMEs predominantly arise as well as their locations at the Sun vary with the solar cycle. During solar minimum, the majority of CMEs originate from the coronal streamer belt while the fraction of CMEs associated with mid- and high-latitude active regions and polar crown filament eruptions increases with the increasing solar activity (Gopalswamy et al., 2003; Zhao and Webb, 2003; Riley et al., 2006). The streamer belt region is a source of CMEs throughout the solar cycle, but when solar activity increases the streamer belt extends to higher latitudes and has a larger tilt with respect to the solar equator (Hoeksema et al., 1991). In contrast, around solar minimum the streamer belt configuration is typically flat and located near the solar equator. During this time, the strong polar coronal fields guide the CMEs that originate from mid- and highlatitude source regions towards the equator (Plunkett et al., 2001; Cremades et al., 2005). During the ascending phase of the solar cycle, the polar field strength weakens and CMEs are deflected less frequently.

The divergence in the ICME and CME rates during the SC 23 minimum reported by Kilpua et al. (2009b) is unexpected. Based on the considerations presented above, the majority of CMEs should propagate close to the ecliptic plane at solar minimum and thus the CME and near-ecliptic ICME rates should track each other, as indicated by Riley et al. (2006) for the SC 22 minimum. It is possible that the divergence during the SC 23 minimum is explained by the unusual solar and heliospheric properties observed during this time. For example, the corona was significantly more complex than a simple dipole that is typically observed at solar minimum conditions, and the polar coronal fields were $40 \%$ weaker than during the three previous minima periods (McComas et al., 2008; Smith and Balogh, 2008; Wang et al., 2009). As a consequence, the low- and mid-latitude coronal holes were frequently observed on the Sun (Luhmann et al., 2009; Wang et al., 2009; Abramenko et al., 2010). This extended period of low solar activity and uncommon solar magnetic field configuration produced interesting consequences throughout the heliosphere. Lee et al. (2009) investigated the effects of the weak polar fields in the ecliptic near-Earth interplanetary medium and they found that the interplanetary magnetic field (IMF), density and momentum flux were lower by $30 \%$, $30 \%$ and $38 \%$, respectively, compared to a similar phase of the SC 22 minimum.

The understanding of how the near-ecliptic ICME rate varies with the solar activity cycle and how it correlates with the CME rate is important for predicting geomagnetic disturbances during different phases of the solar activity cycle (e.g., Gosling et al., 1991; Huttunen et al., 2002), estimating the amount of closed magnetic flux and helicity carried away by the ICMEs (Owens et al., 2007), and estimating how much of the slow solar wind is comprised of transient structures (Kilpua et al., 2009a). Moreover, the examination of how the ICME rate responds to the variations in the CME rate and in the structural changes of the streamer belt will help us to understand the source regions of CMEs and estimate the importance of CME deflection.

The aim of this paper is to study how the near-ecliptic ICME rate responds to the changes in the sunspot number, CME rate and streamer belt structure during the cycles 22 and 23 solar minima. We extend the analysis of Kilpua et al. (2009b) by including observations from the SC 22 minimum period (1995-1997) and from January 2009-April 2010. We will show that streamer belt location experienced significant changes during the SC 23 minimum when the sunspot number and CME activity were very low and had only small variations. Thus, the SC 23 minimum offers a great opportunity to study how changes in the streamer belt structure affect the near-ecliptic ICME rate.

\section{Data and approach}

The time periods selected for this study are from January 1995 through December 1997 for the SC 22 minimum and from January 2007 through April 2010 for the SC 23 minimum.

We use magnetic field and plasma measurements from the near-Earth heliospheric data base (OMNI) at the resolutions of 1- and 5-min. During the 1995-1997 period the OMNI database is created using measurements from Wind, IMP8 and Geotail while during the 2007-2010 period the data comes from Wind and ACE.

Various signatures to distinguish ICMEs from the ambient solar wind have been discussed and summarized by Gosling (1990), Neugebauer and Goldstein (1997), and Zurbuchen and Richardson (2006). Magnetic field signatures of ICMEs include the smooth and organized magnetic field and the enhanced magnetic field magnitude. At $1 \mathrm{AU}$ a large fraction of ICMEs expand, leading to depressed proton temperatures and declining speed profiles. When the speed difference between the ICME and the ambient solar wind is larger than the local magnetosonic speed a fast forward shock is formed ahead of the ICME. Due to the combination of high magnetic field magnitudes and low proton temperatures, ICMEs are typically associated with proton beta (ratio of the proton pressure to the magnetic pressure) values significantly below 1. Magnetic connectivity of ICMEs to the Sun can be studied using observations of suprathermal electrons that carry heat flux away from the Sun along magnetic field lines. Many ICMEs are associated with oppositely flowing (bidirectional) suprathermal electron beams (BDEs), implying that the magnetic structure is attached to the Sun at both ends (e.g., Gosling, 1990).

In this study we investigate the following ICME signatures: enhanced magnetic field magnitude, low magnetic field variance, smooth rotation of the magnetic field direction over a large angle, organization of magnetic field, depressed proton temperature, depressed plasma beta, intervals 
of BDEs, declining solar wind speed and fast forward shocks. For our ICME event selection, we require the ICME intervals to last at least three hours, and that an event includes at least one of the magnetic field signatures and two other features listed above. The ICME boundaries were determined primarily from the magnetic field signature. Since we require a magnetic field signature for an event selection and as some ICMEs may have unorganized magnetic fields, we obtain a lower limit estimation for the ICME rate. However, as demonstrated by Richardson and Cane (2004) at solar minimum, nearly all ICMEs are magnetic clouds. Magnetic clouds form a subset of ICMEs that exhibit enhanced magnetic field magnitude, smooth rotation of the magnetic field direction and low proton beta (Burlaga et al., 1981). The fast forward shocks signatures were identified from the solar wind measurements as simultaneous jumps in magnetic field magnitude and plasma parameters.

Because it is difficult to objectively identify ICMEs, various ICME catalogues have significant differences in the selection of events (Richardson and Cane, 2010). Although there are relatively few wide CMEs during periods of low solar activity, high-quality solar images from $\mathrm{SOHO} / \mathrm{LASCO}$ and STEREO/SECCHI have revealed that narrow CMEs are expelled from the Sun several times per day even at the deepest part of the solar minimum period (Gilbert et al., 2001; Sheeley Jr. et al., 2008). Thus, ICMEs exhibit a vast range of scale sizes. Several studies have indicated that small ICMEs are commonly embedded in the solar wind (Moldwin et al., 2000; Kilpua et al., 2009a; Rouillard et al., 2009). In this study, we consider small and weak ICMEs, and thus our ICME list includes more ICMEs than the list published by Richardson and Cane (2010) for the corresponding period. Our ICME list excludes the events from their list that did not show clear magnetic field signatures.

In Fig. 1 we present an example event of a small, weak ICME that is included in our study but not in the Richardson and Cane (2010) ICME list. During the ICME interval, marked by the blue-shaded region, the magnetic field fluctuation level is depressed with respect to the ambient solar wind. There is a slight rotation in the magnetic field direction (panel b) and a clear depression in proton beta (panel f). The region of low temperature (panel d) starts about one day before the ICME, but the strongest depression in temperature occurs during the ICME interval marked in Fig. 1. The low temperature region before the ICME does not exhibit any other ICME signatures. In Fig. 1d the blue curve represents the measured proton temperature and the red curve represents the "expected proton temperature" $\left(T_{\mathrm{ex}}\right)$ based on the solar wind speed-proton temperature dependence (Richardson and Cane, 1995). Panel 1c shows a declining speed profile (speed difference between the leading edge and the trailing edge of the ICME is $\sim-40 \mathrm{~km} \mathrm{~s}^{-1}$ ). During this ICME interval, suprathermal electrons from the 3-DP instrument onboard the Wind spacecraft (data not shown) indicated a unidirectional heat flux flow, implying that magnetic field

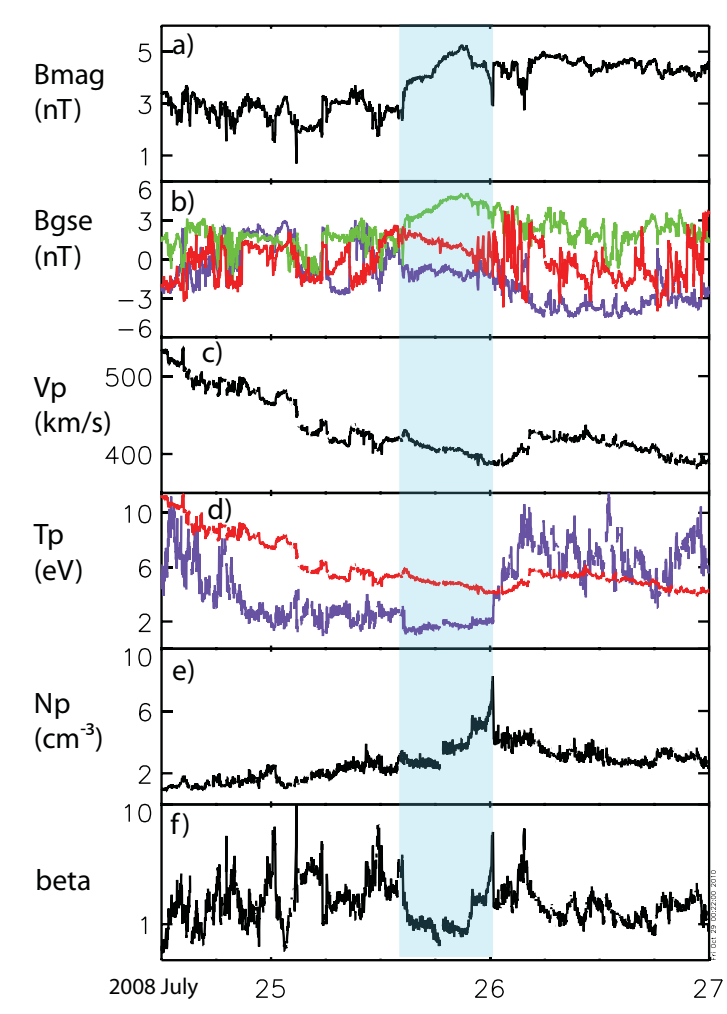

Fig. 1. An ICME (Event SC23/13) identified from the OMNI measurements on 25 July 2008 13:25 UT-25 July 2008 23:30 UT. The blue-shaded area shows the interval of the ICME. The panels show: (a) magnetic field magnitude, (b) magnetic field components in GSE coordinates (blue: $B_{\mathrm{X}}$, green: $B_{\mathrm{Y}}$, red: $B_{\mathrm{Z}}$ ), (c) solar wind speed (d) solar wind proton temperature (blue) and $T_{\mathrm{ex}}$ (red), (e) proton density, and (f) proton beta.

lines within the ICME were attached to the Sun only from the one end. Panel a shows that the maximum magnetic field magnitude of this ICME was only $5.2 \mathrm{nT}$ and its radial width (duration of the ICME times its average speed) was 0.1 AU. These values are significantly smaller than the average maximum magnetic field magnitudes and radial widths typically reported in the ICME lists. For example, the magnetic clouds included in the list by Lepping et al. (2006) have the average maximum magnetic field around $18 \mathrm{nT}$ and the average radial dimensions of about $0.25 \mathrm{AU}$. Although the event is weak, Fig. 1 shows an example of an ICME that stands out from the surrounding solar wind.

\section{ICMEs at solar minimum}

\subsection{Near-ecliptic ICME rate}

We identified 47 ICMEs during the period of 36 months of the SC 22 minimum and 43 ICMEs during the period of 40 months of the SC 23 minimum. Tables 1 and 2 show the time intervals of these ICMEs as well as their maximum 
Table 1. ICMEs identified from the OMNI data base from January 1995 to December 1997. The columns show, the event number, time of the interplanetary shock, start and stop times of the ICME interval, maximum magnetic field within the ICME $\left(B_{\max }\right)$, average speed of the $\operatorname{ICME}\left(V_{\text {ave }}\right)$, and the average width of the ICME ( $\left.D_{\text {ICME }}\right)$.

\begin{tabular}{|c|c|c|c|c|c|c|}
\hline$N$ & Shock (UT) & Start (UT) & Stop (UT) & $B_{\max }(\mathrm{nT})$ & $V_{\text {ave }}\left(\mathrm{km} \mathrm{s}^{-1}\right)$ & $D_{\mathrm{ICME}}(\mathrm{AU})$ \\
\hline \multicolumn{7}{|c|}{1995} \\
\hline 1 & $2 / 719: 30$ & $2 / 804: 10$ & $2 / 903: 45$ & 12.5 & 410 & 0.22 \\
\hline 2 & $3 / 400: 25$ & $3 / 412: 05$ & $3 / 504: 10$ & 12.8 & 444 & 0.17 \\
\hline 3 & & $4 / 301: 10$ & $4 / 415: 45$ & 10.5 & 311 & 0.29 \\
\hline 4 & & 5/13 11:04 & 5/13 17:16 & 14.0 & 331 & 0.11 \\
\hline 5 & & 6/27 09:40 & $6 / 2722: 40$ & 5.3 & 395 & 0.12 \\
\hline 6 & $8 / 17$ 02:52 & $8 / 17$ 13:24 & 8/18 01:48 & 9.3 & 411 & 0.13 \\
\hline 7 & $8 / 22 \quad 12: 50$ & $8 / 22 \quad 19: 30$ & $8 / 2322: 55$ & 11.4 & 359 & 0.25 \\
\hline 8 & & 9/15 18:08 & 9/16 03:10 & 11.9 & 427 & 0.093 \\
\hline 9 & & $9 / 2713: 24$ & $9 / 2721: 32$ & 14.4 & 396 & 0.078 \\
\hline 10 & & 9/29 11:06 & 9/30 06:12 & 7.0 & 361 & 0.17 \\
\hline 11 & & $10 / 321: 44$ & 10/4 03:28 & 11.6 & 406 & 0.056 \\
\hline 12 & 10/18 11:04 & 10/18 19:16 & $10 / 2002: 40$ & 28.7 & 409 & 0.31 \\
\hline 13 & & $10 / 2419: 25$ & $10 / 2507: 52$ & 7.6 & 381 & 0.11 \\
\hline 14 & & $12 / 211: 12$ & $12 / 220: 32$ & 10.6 & 369 & 0.083 \\
\hline 15 & $12 / 1504: 56$ & $12 / 1601: 14$ & 12/16 19:10 & 12.6 & 402 & 0.17 \\
\hline 16 & & 12/31 06:07 & 12/31 10:16 & 11.1 & 379 & 0.038 \\
\hline \multicolumn{7}{|c|}{1996} \\
\hline 17 & & $1 / 122: 15$ & $1 / 207: 11$ & 5.4 & 379 & 0.081 \\
\hline 18 & & $1 / 2004: 54$ & $1 / 2016: 38$ & 11.6 & 472 & 0.13 \\
\hline 19 & & 5/27 15:00 & 5/29 03:32 & 16.0 & 370 & 0.32 \\
\hline 20 & $7 / 113: 25$ & $7 / 120: 28$ & $7 / 211: 44$ & 13.8 & 352 & 0.19 \\
\hline 21 & & $8 / 7$ 10:45 & $8 / 811: 45$ & 8.1 & 346 & 0.21 \\
\hline 22 & & $12 / 1316: 45$ & $12 / 14$ 08:40 & 6.0 & 432 & 0.17 \\
\hline 23 & & $12 / 2402: 50$ & $12 / 2512: 00$ & 12.7 & 349 & 0.29 \\
\hline \multicolumn{7}{|c|}{1997} \\
\hline 24 & 1/10 00:46 & $1 / 1004: 50$ & 1/11 05:06 & 22.3 & 438 & 0.30 \\
\hline 25 & $2 / 913: 20$ & $2 / 1003: 10$ & 2/10 19:14 & 9.0 & 463 & 0.18 \\
\hline 26 & $2 / 2718: 14$ & $2 / 2720: 39$ & $2 / 2723: 42$ & 14.0 & 514 & 0.037 \\
\hline 27 & & 3/25 14:06 & $3 / 2517: 27$ & 8.7 & 412 & 0.033 \\
\hline 28 & & 4/11 06:20 & 4/11 20:26 & 22.5 & 462 & 0.16 \\
\hline 29 & & 4/21 13:00 & 4/23 11:35 & 13.9 & 363 & 0.41 \\
\hline 30 & $5 / 15$ 01:36 & 5/15 08:26 & 5/16 00:40 & 25.3 & 455 & 0.18 \\
\hline 31 & 5/20 06:40 & $5 / 20 \quad 15: 34$ & 5/21 05:46 & 7.6 & 286 & 0.098 \\
\hline 32 & 5/26 09:55 & $5 / 26 \quad 16: 40$ & 5/28 01:10 & 11.0 & 330 & 0.26 \\
\hline 33 & & $6 / 19$ 05:20 & $6 / 2000: 40$ & 8.6 & 350 & 0.16 \\
\hline 34 & & $>7 / 913: 36$ & 7/9 20:18 & 13.9 & 356 & $? ?$ \\
\hline 35 & 7/15 03:02 & 7/15 09:22 & 7/16 11:22 & 12.4 & 357 & 0.22 \\
\hline 36 & & $8 / 314: 26$ & $8 / 402: 28$ & 16.7 & 438 & 0.13 \\
\hline 37 & & $9 / 102: 26$ & $9 / 115: 36$ & 4.3 & 376 & 0.12 \\
\hline 38 & $9 / 222: 56$ & $9 / 3$ 13:00 & $9 / 320: 50$ & 17.3 & 406 & 0.077 \\
\hline 39 & & 9/18 04:25 & $9 / 2012: 15$ & 12.9 & 321 & 0.43 \\
\hline 40 & & $9 / 2122: 18$ & 9/22 18:04 & 18.3 & 425 & 0.20 \\
\hline 41 & 10/1 00:20 & $10 / 1 \quad 15: 54$ & 10/2 23:05 & 10.5 & 453 & 0.34 \\
\hline 42 & & $10 / 1021: 10$ & 10/12 03:12 & 13.6 & 400 & 0.29 \\
\hline 43 & & $10 / 2710: 26$ & $10 / 2805: 50$ & 8.7 & 463 & 0.22 \\
\hline 44 & $11 / 622: 40$ & $11 / 07$ 05:50 & $11 / 815: 38$ & 18.0 & 420 & 0.34 \\
\hline 45 & 11/22 10:00 & $11 / 22$ 15:08 & $11 / 2313: 06$ & 27.1 & 500 & 0.27 \\
\hline 46 & $12 / 1005: 36$ & $12 / 1019: 32$ & 12/11 11:34 & 16.0 & 358 & 0.14 \\
\hline 47 & $12 / 3002: 14$ & $12 / 3010: 58$ & 12/31 07:44 & 13.9 & 367 & 0.18 \\
\hline
\end{tabular}


Table 2. ICMEs identified from the OMNI data base from January 2007 to April 2010. The columns are same as in Table 1.

\begin{tabular}{|c|c|c|c|c|c|c|}
\hline$N$ & Shock (UT) & Start (UT) & Stop (UT) & $B_{\max }(\mathrm{nT})$ & $V_{\text {ave }}\left(\mathrm{km} \mathrm{s}^{-1}\right)$ & $D_{\text {ICME }}(\mathrm{AU})$ \\
\hline \multicolumn{7}{|c|}{2007} \\
\hline 1 & & 1/14 11:45 & $1 / 1507: 45$ & 15.2 & 358 & 0.17 \\
\hline 2 & & 1/15 20:50 & $1 / 1604: 45$ & 10.1 & 518 & 0.10 \\
\hline 3 & & $2 / 204: 10$ & $2 / 214: 35$ & 3.7 & 514 & 0.13 \\
\hline 4 & & $3 / 2405: 15$ & $3 / 24$ 12:30 & 10.9 & 364 & 0.064 \\
\hline 5 & & 3/29 15:05 & 3/30 00:20 & 6.2 & 396 & 0.088 \\
\hline 6 & & $5 / 2122: 45$ & $5 / 22 \quad 15: 55$ & 11.8 & 445 & 0.18 \\
\hline 7 & & $6 / 805: 45$ & 6/9 05:15 & 9.6 & 362 & 0.21 \\
\hline 8 & & 6/30 00:15 & $7 / 100: 45$ & 8.9 & 502 & 0.29 \\
\hline 9 & & 7/23 21:00 & 7/24 09:45 & 3.2 & 377 & 0.12 \\
\hline 10 & $11 / 19$ 17:23 & $11 / 2000: 45$ & $11 / 2011: 50$ & 14.6 & 462 & 0.12 \\
\hline 11 & & $12 / 2515: 15$ & 12/26 10:00 & 6.2 & 354 & 0.16 \\
\hline \multicolumn{7}{|c|}{2008} \\
\hline 12 & & $3 / 817: 45$ & $3 / 904: 40$ & 14.5 & 452 & 0.12 \\
\hline 13 & & 7/25 13:25 & $7 / 25$ 23:30 & 5.2 & 408 & 0.10 \\
\hline 14 & & 7/31 11:49 & 7/31 15:29 & 7.1 & 380 & 0.033 \\
\hline 15 & & $9 / 316: 30$ & 9/4 03:45 & 13.5 & 461 & 0.12 \\
\hline 16 & $9 / 1612: 33$ & 9/17 04:00 & 9/18 08:00 & 7.2 & 410 & 0.28 \\
\hline 17 & & 10/8 04:45 & 10/8 20:15 & 5.5 & 353 & 0.13 \\
\hline 18 & & 11/1 07:30 & $11 / 115: 30$ & 5.3 & 491 & 0.094 \\
\hline 19 & & $12 / 418: 15$ & 12/5 12:00 & 7.3 & 386 & 0.17 \\
\hline 20 & $12 / 1616: 40$ & 12/17 03:30 & $12 / 1714: 30$ & 9.8 & 343 & 0.09 \\
\hline \multicolumn{7}{|c|}{2009} \\
\hline 21 & & 1/14 12:10 & 1/15 06:15 & 8.1 & 346 & 0.15 \\
\hline 22 & & 1/19 01:25 & 1/19 05:10 & 13.4 & 430 & 0.04 \\
\hline 23 & $2 / 319: 20$ & $2 / 400: 10$ & $2 / 417: 45$ & 11.2 & 355 & 0.15 \\
\hline 24 & $3 / 1122: 26$ & 3/12 01:22 & 3/13 03:42 & 18.6 & 365 & 0.23 \\
\hline 25 & & 4/8 01:44 & 4/8 13:06 & 3.6 & 344 & 0.094 \\
\hline 26 & & 4/22 14:14 & $4 / 2220: 44$ & 4.4 & 390 & 0.061 \\
\hline 27 & $5 / 2809: 58$ & 5/28 09:58 & $5 / 2907: 56$ & 8.6 & 413 & 0.22 \\
\hline 28 & & $6 / 27$ 15:20 & $6 / 2820: 30$ & 12.2 & 390 & 0.27 \\
\hline 29 & & $7 / 2102: 14$ & $7 / 22$ 03:38 & 14.5 & 321 & 0.20 \\
\hline 30 & & $8 / 511: 14$ & $8 / 606: 34$ & 14.6 & 375 & 0.17 \\
\hline 31 & & $8 / 3008: 36$ & $8 / 3014: 46$ & 12.7 & 401 & 0.06 \\
\hline 32 & 9/30 01:40 & 9/30 06:34 & 9/30 19:24 & 9.3 & 347 & 0.12 \\
\hline 33 & & 10/17 17:30 & 10/18 13:40 & 3.7 & 316 & 0.15 \\
\hline 34 & & 10/29 03:08 & $10 / 3010: 50$ & 11.3 & 362 & 0.28 \\
\hline 35 & & 11/14 11:55 & $11 / 1514: 15$ & 8.4 & 335 & 0.21 \\
\hline 36 & & $11 / 2018: 40$ & 11/21 02:06 & 11.3 & 415 & 0.074 \\
\hline 37 & $12 / 505: 25$ & $12 / 602: 44$ & 12/6 21:00 & 9.7 & 391 & 0.17 \\
\hline 38 & 12/12 04:39 & 12/12 22:05 & $12 / 13$ 23:45 & 8.2 & 271 & 0.17 \\
\hline \multicolumn{7}{|c|}{2010} \\
\hline 39 & & $1 / 123: 10$ & 1/3 13:20 & 8.8 & 287 & 0.26 \\
\hline 40 & $2 / 718: 10$ & 2/8 00:00 & $2 / 907: 30$ & 10.7 & 355 & 0.27 \\
\hline 41 & & $2 / 2513: 15$ & $2 / 2520: 20$ & 7.1 & 357 & 0.063 \\
\hline 42 & $4 / 508: 35$ & $4 / 5$ 12:40 & 4/6 14:00 & 19.1 & 643 & 0.40 \\
\hline 43 & 4/11 13:15 & 4/11 21:30 & 4/12 16:20 & 12.1 & 412 & 0.19 \\
\hline
\end{tabular}




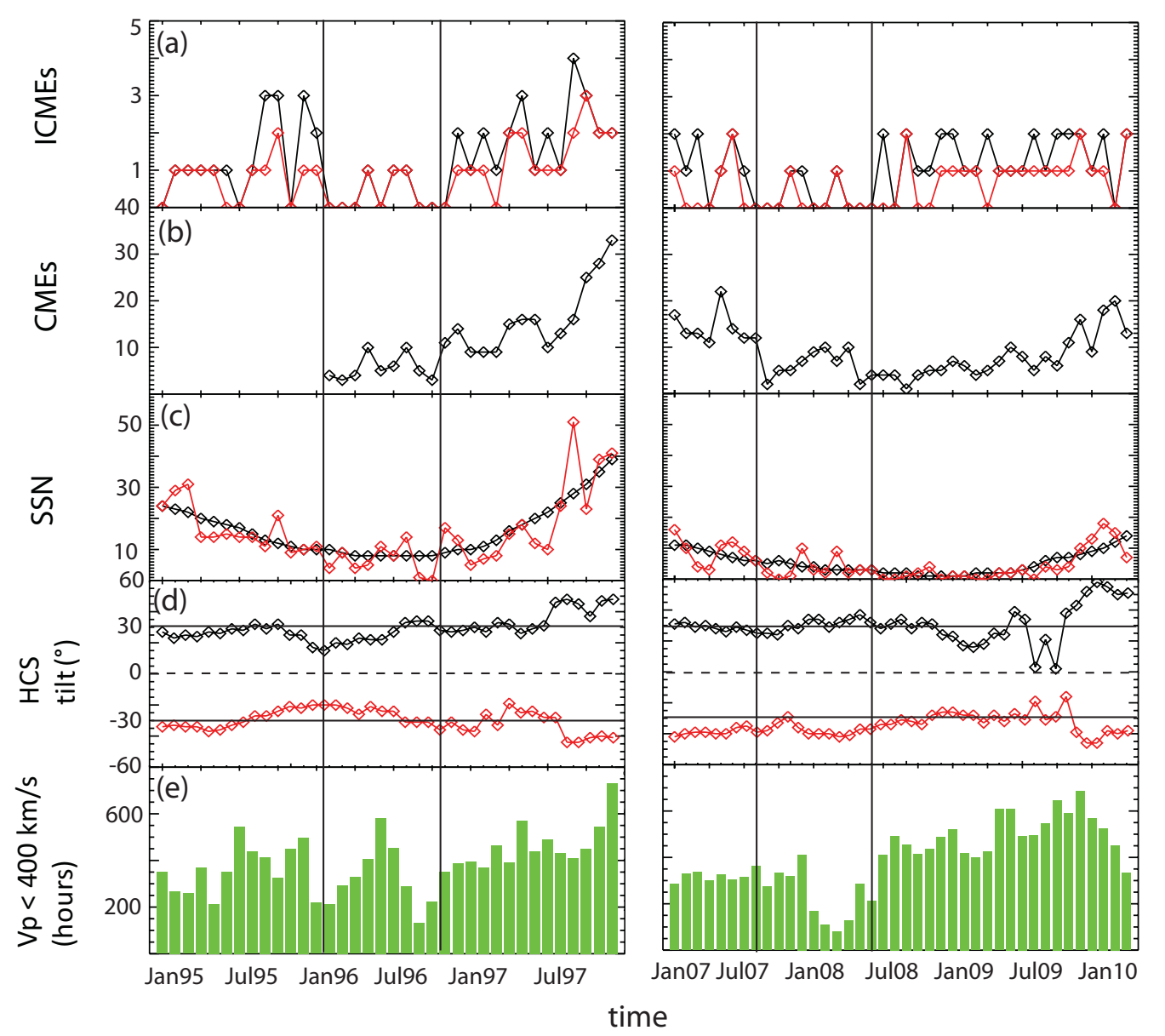

Fig. 2. Monthly ICME rate and solar activity. Panels show: rate of (a) ICMEs (black) and the subset of ICMEs that have $B_{\mathrm{max}}>7 \mathrm{nT}$ and $D_{\text {ICME }}>0.1 \mathrm{AU}$ (red) (b) CMEs with angular extent $>50^{\circ}$ from the LASCO CME catalogue, (c) monthly (red) and monthly smoothed (black) sunspot number from SIDC, (d) maximum northern (black) and southern (red) latitudinal extent of the streamer belt neutral line calculated using source surface at $1.8 R_{\mathrm{S}}$ in the PFSS model, (e) monthly distributions of periods (in hours) with solar wind speed $<400 \mathrm{~km} \mathrm{~s}^{-1}$. The periods of the minimum ICME rates are bounded between two vertical lines.

magnetic field $\left(B_{\max }\right)$, average solar wind speed $\left(V_{\text {ave }}\right)$ and radial width $\left(D_{\mathrm{ICME}}\right)$. $D_{\mathrm{ICME}}$ is obtained by multiplying the duration of the ICME event with its average speed.

Figure 2 shows observations for the SC 22 minimum (lefthand panel) and the SC 23 minimum (right-hand panel). In each case, panel a shows the monthly ICME rate from the near-Earth solar wind. The black curve shows the rate of all ICMEs included in Tables 1 and 2 while the red curve shows ICMEs that have $B_{\max }>7 \mathrm{nT}$ and $D_{\mathrm{ICME}}$ over $0.1 \mathrm{AU}$. The monthly CME rate is given in the panel b. We include from the online LASCO CME catalogue the events that have angular widths $(\mathrm{AW})>50^{\circ}$. (Note that LASCO CME observations are only available following the launch of SOHO in December 1995). To get a better agreement with the LASCO catalogue and the automated CACTUS catalogue, the identification criteria has changed in the LASCO CME catalogue over the years to include smaller events. For CMEs with angular width greater than few tens of degrees, the CME rate matches much better (Yashiro et al., 2008). The choice of angular width $>50^{\circ}$ is based on these concerns. We point out that small and weak ICMEs included in our statistics could be associated with CMEs narrower than AW $>50^{\circ}$. Panel $\mathrm{c}$ shows the monthly (red) and monthly smoothed (black) sunspot numbers from the Solar Influences Data Center (SIDC). Panel d shows the maximum northern (black) and southern (red) latitudinal extents of the streamer belt neutral line for each Carrington rotation within our study period. To guide the eye we mark the latitudes $\pm 30^{\circ}$ with solid horizontal lines and $0^{\circ}$ latitude with dashed horizontal lines. Note that we include hemispherical information since the northern and southern extents of the streamer belt can differ greatly on occasions. Panel e shows the monthly periods in hours when the solar wind speed was below $400 \mathrm{~km} \mathrm{~s}^{-1}$. The pairs of vertical lines in Fig. 2 delimit the periods when the lowest number of ICMEs were identified. 

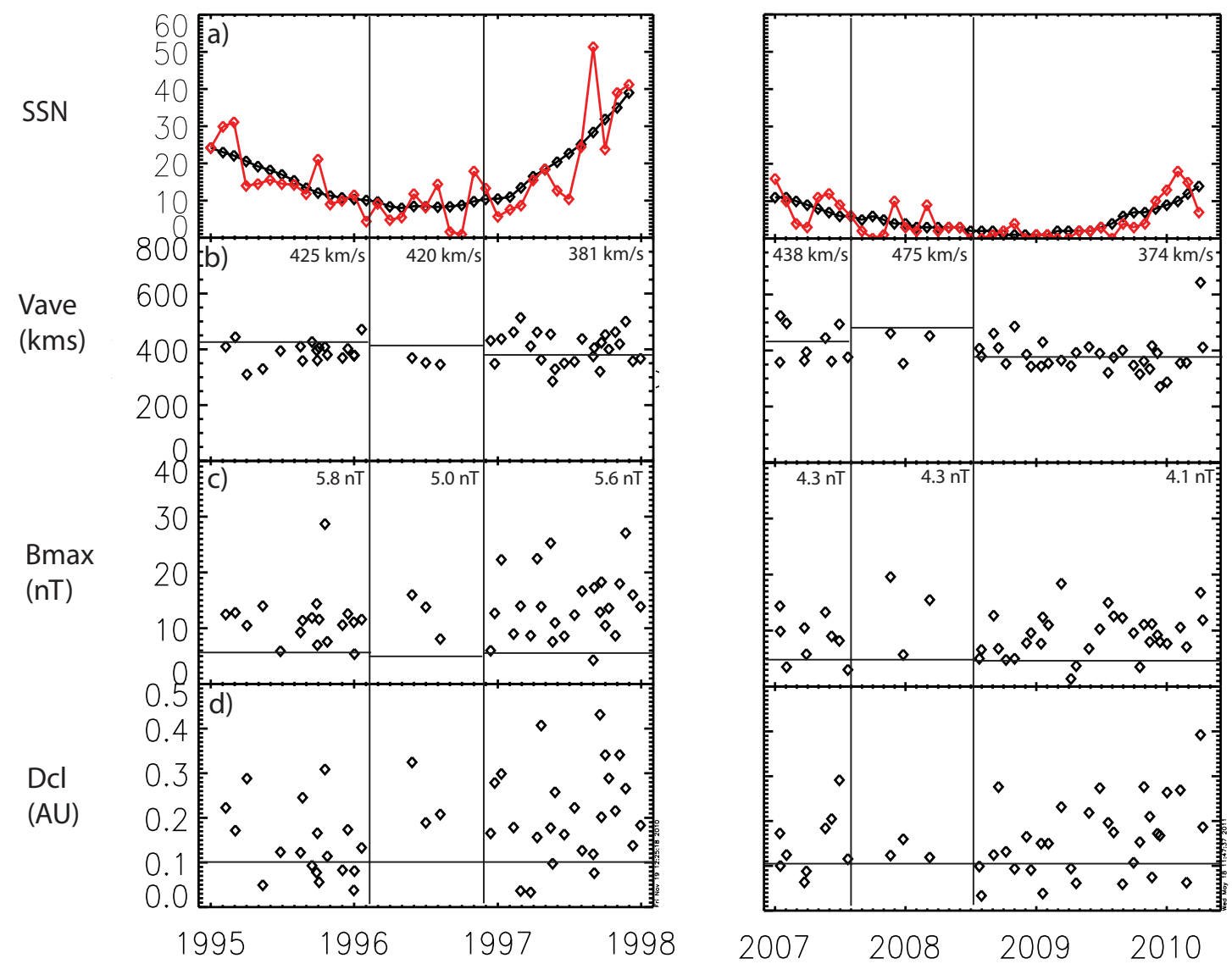

Fig. 3. ICME parameters for the SC 22 (left) and SC 23 (right) minima as a function of time. The panels show from top to bottom: (a) monthly (red) and smoothed (black) monthly sunspot numbers from the Solar Influences Data Center, (b) average solar wind speed, (c) maximum magnetic field, and (d) radial width for each ICME. The period of the lowest ICME rate is bounded between two vertical lines (see Sect. 3.1 and Fig. 2). In panels (b) and (d) the mean solar wind speeds and magnetic field magnitudes averaged over the time periods before, during and after the ICME rate minimum are indicated with horizontal lines and the corresponding values are given above. In panel (d) the horizontal line shows the radial width $0.1 \mathrm{AU}$.

During the SC 22 minimum (left panels of Fig. 2) the monthly ICME rate decreased sharply in February 1996 and remained low for the next 10 months. Within the period February-November 1996 the average ICME rate was 0.3 per month and the average CME rate 6.1 per month. The average smoothed sunspot number was 8.8. From December 1996 to the end of our study period for SC 22, the ICME rate increased to 2.0 per month. In addition, the CME rate and the sunspot number increased steadily from December 1996 to December 1997. The strongest increases in the sunspot number and the CME rate occurred from August 1997 to December 1997. At this time the ICME rate also showed an increasing trend.

From August 2007 to June 2008 of SC 23 (right panels of Fig. 2) the ICME rate was 0.27 per month and the average smoothed sunspot number was 4.5. In comparison, the average CME rate was approximately the same (6.6 per month) as during the time of the lowest ICME rate for SC 22. In July 2008 the ICME rate increased and stayed at a steady level (on average 1.4 per month) for the rest of our SC 23 study period. However, from July 2008 the sunspot number and the CME rate decreased, but increased again during the last six months of our study period (November 2009-April 2010). There was no further increase in the ICME rate observed at this time. As shown in Fig. 2e, in SC 23 during the time of the lowest ICME rate the fraction of slow solar wind speed decreased drastically (from about $300 \mathrm{~h}$ per month to about $100 \mathrm{~h}$ per month). For SC 22 the fraction of slow solar wind speed decreased at the beginning and end of the low ICME rate period, but otherwise the amount of slow solar wind was comparable to the other periods investigated (see also mean solar wind speeds shown in Fig. 3b).

\subsection{Average ICME properties}

Figure $3 \mathrm{~b}$, c, and d show $B_{\max }, V_{\text {ave }}$ and $D_{\text {ICME }}$ plotted as a function of time for the SC 22 (left) and SC $23 \mathrm{~min}$ ima (right). Panels $\mathrm{b}$ and $\mathrm{c}$ also show median values of 
Table 3. Averages of ICMEs during the SC 22 and SC 23 minima. Median values are given in parenthesis.

\begin{tabular}{lll}
\hline & SC 22 minimum & SC 23 minimum \\
\hline$B_{\max }(\mathrm{nT})$ & $13.5(12.5)$ & $9.4(9.2)$ \\
$V_{\text {ave }}\left(\mathrm{km} \mathrm{s}^{-1}\right)$ & $400(399)$ & $394(380)$ \\
$D_{\text {ICME }}(\mathrm{AU})$ & $0.19(0.17)$ & $0.16(0.15)$ \\
\hline
\end{tabular}

solar wind speed and magnetic field averaged over time periods before, during and after the ICME rate minimum period. Panel a gives the monthly (red) and smoothed (black) monthly sunspot numbers from SIDC. Table 3 lists the averages and medians of $B_{\max }, V_{\text {ave }}$ and $D_{\mathrm{ICME}}$ over all ICMEs from the SC 22 and 23 minima.

It is evident that the majority of the observed ICMEs are slow for both SC 22 and 23 minima with $V_{\text {ave }}=400 \pm$ $51 \mathrm{~km} \mathrm{~s}^{-1}$ and $394 \pm 64 \mathrm{~km} \mathrm{~s}^{-1}$, respectively. As shown in Fig. $3 \mathrm{~b}$ the ICME speeds are comparable with the average solar wind speed. We identified two fast ICMEs ( $V_{\text {ave }}$ over $500 \mathrm{~km} \mathrm{~s}^{-1}$ ) for the SC 22 period and four for the SC 23 period.

During the SC 23 minimum the ICMEs have weaker magnetic field magnitudes than during the SC 22 minimum, the averages (standard deviations) of $B_{\max }$ being $9.4 \pm 4.2 \mathrm{nT}$ and $13.5 \pm 5.6 \mathrm{nT}$ for SC 23 and SC 22, respectively. Figure $3 \mathrm{c}$ shows the average magnetic field magnitude was also lower for SC 23 than for SC 22. For both solar cycle periods the majority of ICMEs had $B_{\max }$ above the mean magnetic field magnitude. For the SC 23 period, 13 (31\%) of the identified ICMEs have $B_{\max }$ below $7 \mathrm{nT}$, while for the SC 22 period this was the case only for three ICMEs. For SC 22 the highest $B_{\max }$ values were observed during the later part of our study period, but in general for both solar cycle minimum periods $B_{\max }$ varied over a large range of values.

The scale sizes of ICMEs also vary over a large range of values. The smallest events have $D_{\mathrm{ICME}}$ about $0.03 \mathrm{AU}$ (note that we did not include the events with duration $<3 \mathrm{~h}$ ) and the largest ICMEs have the radial widths about $0.4 \mathrm{AU}$. The SC 23 ICMEs were slightly smaller than the SC 22 ICMEs, with the average values of (standard deviation) $0.16 \mathrm{AU}$ (0.079 AU) and 0.19 AU (0.1 AU) for SC 23 and SC 22, respectively. No clear temporal variation was identified for $D_{\text {ICME}}$.

\section{Solar sources of ICMEs}

\section{Streamer belt development}

In this section we investigate how the large-scale coronal field evolved over the investigated periods. Figures 4 and 5 show synoptic maps for the neutral line of the heliospheric current sheet (HCS) for a selection of Carrington rotations during the SC 22 and SC 23 minima. The HCS neutral line is calculated from the Mount Wilson Observatory (MWO) and GONG photospheric field synoptic maps using the potential field source surface (PFSS) model of the coronal magnetic field (Altschuler and Newkirk, 1969; Schatten et al., 1969). Note that we use the value of 1.8 solar radii for the source surface radius in the model rather than the canonical value of $2.5 R_{\mathrm{S}}$ that is typically used in potential field applications. As Lee et al. (2011) recently demonstrated, the $1.8 R_{\mathrm{S}}$ source surface radius in the PFFS model produces a more consistent picture of both the solar sources and the IMF for the SC 23 minimum period. For the SC 22 minimum period a slightly larger value $\left(\sim 1.9 R_{\mathrm{S}}\right)$ was determined.

Figures 4 and $2 \mathrm{~d}$ left show that the neutral line had a high inclination configuration during 1995 but became flatter and shifted closer to the solar equator by solar minimum in 1996 (see also Sanderson et al., 1998). During 1997 when the solar activity and the ICME rate increased, the neutral line became more warped and shifted to higher latitudes. The most dramatic transition is seen in August 1997 (see Fig. 2d left) when both the northern and southern latitudinal extents of the neutral line suddenly shifted to higher latitudes. This transition coincided with clear increases in the CME rate and in the sunspot number.

Figures $2 d$ right and 5 demonstrate that during SC 23 the streamer belt neutral line was located at higher latitudes and was more warped than during SC 22 (see also Sanderson et al., 1998; Lee et al., 2010). One difference is that for the SC 23 minimum the neutral line experienced significant warps while the sunspot number and the CME activity remained very low and had only small variations. In addition, during the period when the ICME rate was at its lowest level (August 2007-July 2008) the neutral line extended beyond $\pm 30^{\circ}$ and maintained a warped configuration (see also Lee et al., 2010). The neutral line was located closest to the equator and had the flattest structure after the lowest ICME rate period. Figure 2d right shows that in November 2009 the neutral line suddenly shifted to higher latitudes. During the last few months of our SC 23 study period, the structure was complex and highly warped. This transition was accompanied with increases in the CME rate and sunspot number, but there was no increase in the ICME rate.

\section{Discussion and summary}

In this paper we have performed a statistical study of the occurrence rates and properties of ICMEs and their connection to the variations in the solar activity and streamer belt configuration during solar minimum conditions following solar cycles 22 and 23. The ICMEs were identified from the nearEarth spacecraft measurements close to the ecliptic plane. The SC 23 minimum was in many respects different from the corresponding phase of the previous solar cycle (e.g., McComas et al., 2008; Smith and Balogh, 2008; Lee et al., 2009, 

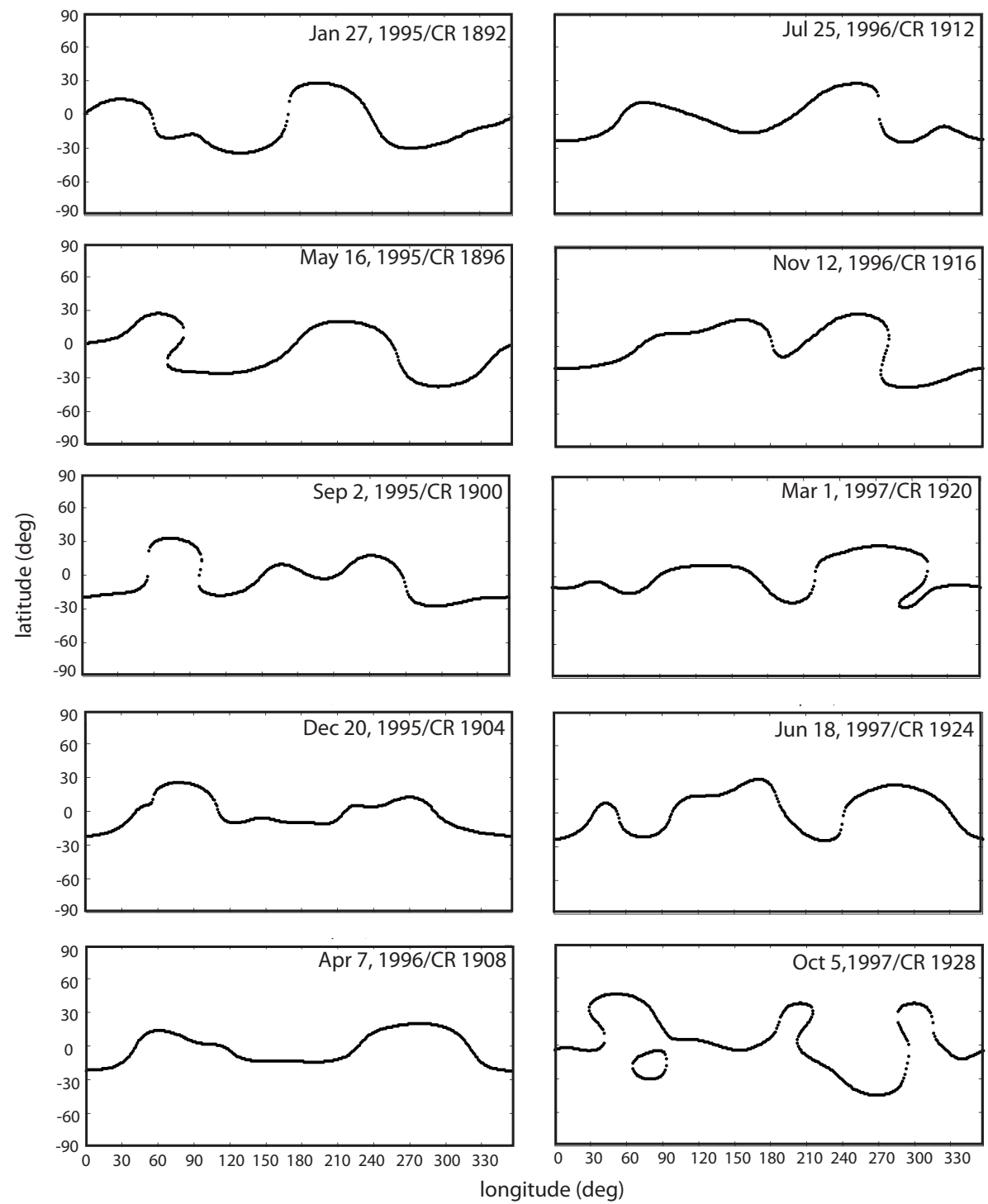

Fig. 4. Source surface synoptic maps from the Mount Wilson Observatory for selected Carrington rotations during the SC 22 minima. The black line indicates the neutral line. The date indicated gives the start time of each Carrington rotation.

2011; Luhmann et al., 2009; Abramenko et al., 2010). We demonstrated in this paper that there were differences in the general ICME properties between the SC 23 minimum and the previous minimum.

The maximum magnetic fields of the SC 23 ICMEs were $\sim 30 \%$ lower and their radial widths were $\sim 15 \%$ lower compared to the SC 22 ICMEs. The low magnetic field magnitude and small-scale size of ICMEs can be due to its intrinsic properties or from the spacecraft encountering the ICME close to the outer boundary. In a case when the spacecraft makes only a glancing encounter through the ICME the magnetic field magnitude is lower and the duration of the ICME is shorter than when the spacecraft encounters the ICME centrally. The analysis of CME mass and energy properties over a full solar cycle (1996-2009) by Vourlidas et al. (2010, 2011) (see Fig. 1 in Vourlidas et al., 2011) indicates that during the SC 23 minimum CMEs had clearly lower masses, speeds and kinetic energies than in the previous minimum. As discussed in Sect. 1 also coronal and heliospheric magnetic fields were significantly lower during the SC 23 minimum than during a few previous solar cycles (Wang et al., 2009; Lee et al., 2009). In addition, the warped and high-latitude streamer belt configuration observed during the SC 23 minimum (Sect. 4.1) suggests that the CMEs were launched frequently from mid- and high-heliolatitudes. As a consequence, there may have been a significant ICME population near the ecliptic plane that were encountered far from the center. 

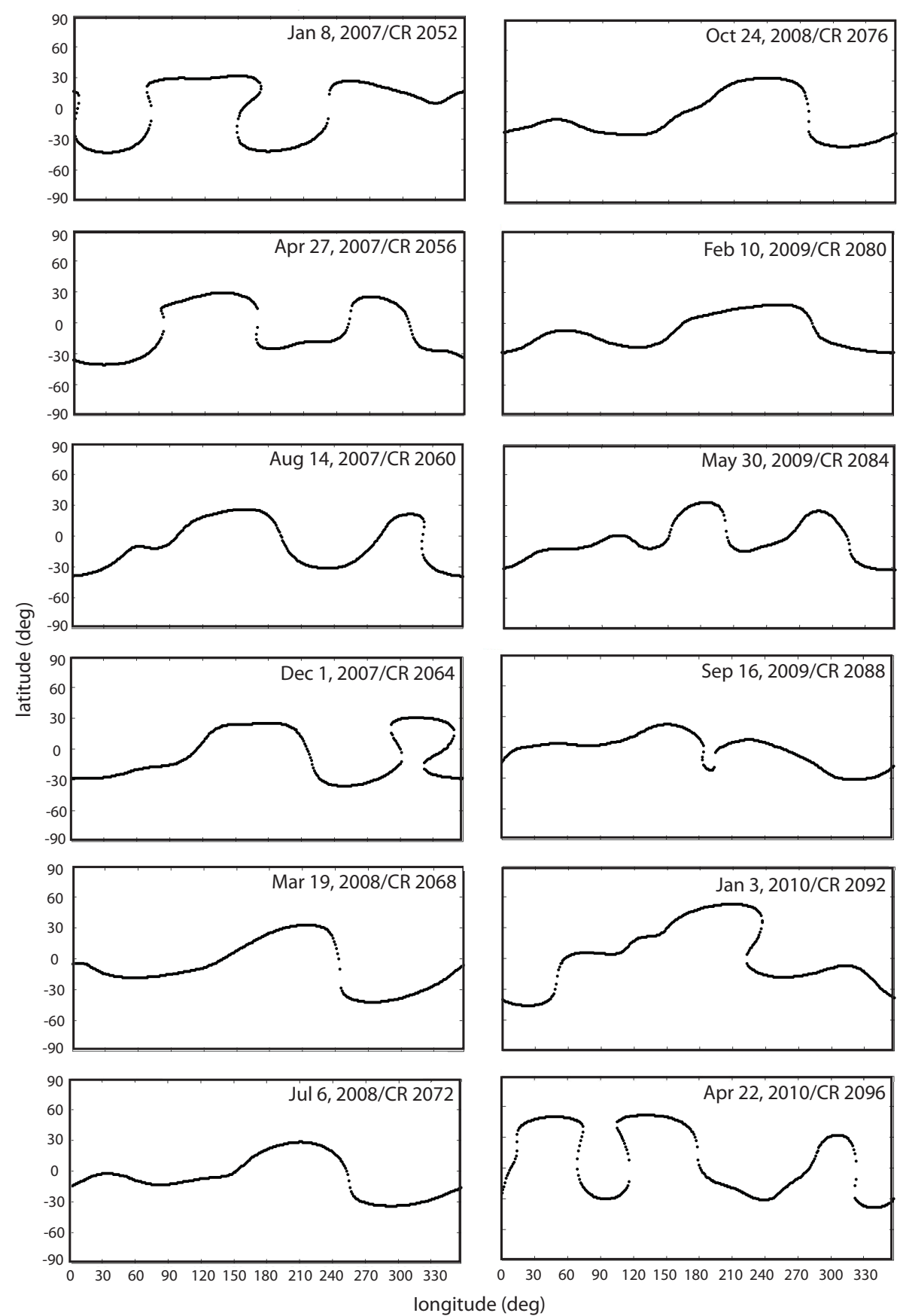

Fig. 5. Source surface synoptic maps from the Mount Wilson Observatory and GONG for selected Carrington rotations during the SC 23 minima. The black line indicates the neutral line. The date indicated give the start time of each Carrington rotation.

Previous studies have suggested that the streamer belt region is a primary source of CMEs near solar minimum (e.g., Zhao and Webb, 2003; Riley et al., 2006). As discussed in Sect. 1 the fraction of CMEs that propagate along the ecliptic is correlated with the configuration of the streamer belt neutral line. Our results show that during a "typical" solar minimum, such as the SC 22 minimum, the variations in the CME rate are large enough to control the variations in the near-ecliptic ICME rate. We found that for SC 22 the near-ecliptic ICME rate, CME rate and sunspot number roughly tracked each other throughout the solar minimum period (see also Riley et al., 2006). The minimum ICME rate of SC 22 coincided with the period when the global solar magnetic field was approximately a simple dipole configuration, featured by the flat and low-latitude streamer belt. Riley et al. (2006) showed that divergence in CME and ICME rates 
did not occur until 1998, after the period considered in the current study. They suggested that this divergence during the ascending phase of SC 23 can be explained by an increased number of CMEs originating from mid-latitude active regions and polar crown filament eruptions.

In contrast, for the SC 23 minimum, the effect of the streamer belt structural changes seemed to affect the nearecliptic ICME rate throughout the solar minimum period. During the SC 23 minimum streamer belt was clearly more warped than during the SC 22 minimum and became flat for only a few months in late 2009 (see also Lee et al., 2010). At this time the sunspot number and CME rate already showed an increasing trend. During our SC 23 study period the number of CMEs correlated with the sunspot number, but the variations in the ICME rate did not follow the changes in the CME rate.

For SC 23 when the ICME rate was at its lowest level (August 2007-June 2008), the streamer belt was highly warped and located at relatively high latitudes. The warped streamer belt configuration gave rise to pseudostreamers (Wang et al., 2007) that separated coronal holes of the same polarity and where CMEs frequently occur. At this time the fraction of slow speed solar wind near the ecliptic decreased drastically. This decrease was presumably due to the prevalence of high-speed solar wind from the low- and mid-latitude coronal holes (Lee et al., 2009; Abramenko et al., 2010). Thus, the CME source regions were located primarily at mid- and high-latitudes. Due to the presence of low-latitude coronal holes, the CMEs could not be guided towards the equator.

In mid-2008 the ICME rate increased although the CME rate and sunspot number further declined. At this time the streamer belt started to develop towards a flatter configuration. The fraction of slow speed solar wind near the ecliptic increased considerably, indicating that the mid- and highlatitude CMEs could be effectively channelled towards the equator. Because the polar coronal fields were atypically weak during the SC 23 minimum, we propose that the CME deflection at that time is mainly due to kinematic effects. The slow and weak CMEs are essentially convected with the solar wind to low latitudes, in contrast to the bigger and faster CMEs that can travel through the solar wind more radially (Xie et al., 2009; Kilpua et al., 2009b). During the SC 23 minimum the average CME masses and speeds were lower than in the previous minimum (Vourlidas et al., 2011), making the kinematic deflection a likely scenario. From the end of 2009 the CME rate and sunspot number started to increase, but no increase in the number of ICMEs was observed. This lack of increase in the ICME rate can be attributed to the streamer belt becoming highly warped and shifting the CME source regions to higher heliolatitudes.

During the period of the lowest ICME rate for both SC 22 and SC 23 minima, the number of observed ICMEs was about one ICME every three months, consistent with the minimum ICME rates reported in previous studies (e.g., Richardson and Cane, 2010). Our results show that during the deep- est minimum of SC 23 based on sunspot numbers, the ICME rate was more than four times higher than the minimum rate of 0.3 per month. As discussed above, this relatively high ICME rate did not result from the increased CME rate, but from the increased number of CMEs propagating close to the ecliptic. It should be noted that due to low solar activity we have very limited statistics and caution should be exercised when drawing conclusions from them.

Our analysis shows that even during this deep solar minimum of SC 23 the ICMEs are regularly embedded in the solar wind. Based on our selection criteria (see Sect. 2), we obtained a lower limit estimation of the ICME rate. The presence of ICMEs can modulate the cosmic ray intensity at $1 \mathrm{AU}$ (Cane, 2000) and affect to the structure of the co-rotating interaction regions (CIRs) and consequently to the CIR associated solar energetic particle events (Gomez-Herrero et al., 2010). The increased number of ecliptic ICMEs may also enhance magnetospheric activity at the Earth, although near solar minimum the magnetosheric disturbances associated with ICMEs are mainly weak or moderate. Because the ICME rate sampled near the ecliptic plane does not necessarily correlate with the total number of CMEs, one should be careful when estimating magnetic flux and the helicity (Qiu et al., 2007) removed from the Sun based on the nearecliptic ICME rate. In addition, it can make a large difference whether small and weak ICMEs represent intrinsically weak CMEs or larger CMEs encountered close to edge. In the latter case, the magnetic flux content of an ICME can be significantly underestimated. Our results suggest that, at least for the SC 23 minimum, weak and small CMEs originated from both weak CMEs and from larger CMEs sampled far from the center. The spacecraft trajectory through the ICMEs could be estimated using advanced ICME modeling methods, such as Grad-Shafranov reconstruction (Hu and Sonnerup, 2002), which will be left for future work.

Acknowledgements. The sunspot data were archived through SIDC-team, World Data Center for the Sunspot Index, Royal Observatory of Belgium. We acknowledge National Geophysical Data Center for providing the OMNI data, Mount Wilson Observatory and the Global Oscillation Network Group (GONG) for providing access to their magnetogram data sets. The LASCO CME catalogue is generated and maintained at the CDAW Data Center by NASA and The Catholic University of America in cooperation with the Naval Research Laboratory. SOHO is a project of international cooperation between ESA and NASA. Y. Li acknowledges support from NASA GI fund NNX08AJ04G and NSF CISM fund ATM-0120950. E. Kilpua acknowledges Academy of Finland (project 130298) for financial support.

Topical Editor R. Forsyth thanks I. Richardson for his help in evaluating this paper. 


\section{References}

Abramenko, V., Yurchyshyn, V., Linker, J., Mikic, Luhmann, J., and Lee, C. O.: Low latitude coronal holes at the minimum of the 23rd solar cycle, 712, 813-818, Astrophys. J., 2010.

Altschuler, M. D. and Newkirk, G.: Magnetic fields and the structure of the solar corona. I: methods of calculating coronal fields, Sol. Phys., 9, 131-149, 1969.

Burlaga, L., Sittler, E., Mariani, F., and Schwenn, R.: Magnetic loop behind an interplanetary shock: Voyager, Helios and IMP 8 observations, J. Geophys. Res., 86, 6673-6684, 1981.

Cane, H. V.: Coronal mass ejections and Forbush decreases, Space Sci. Rev., 93, 55-77, 2000.

Cremades, H., Bothmer, V., and Tripathi, D.: Properties of structured coronal mass ejections in solar cycle 23, Adv. Space Res., 38, 461-465, doi:10.1016/j.asr.2005.01.095, 2005.

Gilbert, H. R., Serex, E. C, Holzer ,T. E., MacQueen, R. M., and McIntosh, P. S.: Narrow coronal mass ejections, Astrophys. J., 550, 1093-1101, 2001.

Gomez-Herrero, R., Malandraki, O. E., Dresing, N., Kilpua, K., Heber, B., Klassen, A., Muller-Mellin, R., and WimmerSchweingruber, R. F.: Spatial and temporal variations of CIRs: multi-point observations by STEREO, J. Atmos. Sol.-Terr. Phy., 73, 551-565, doi:10.1016/j.jastp.2010.11.017, 2011.

Gopalswamy, N., Lara, A., Yashiro, S., and Howard, R. A.: Coronal mass ejections and solar polarity reversal, Astrophys. J., 598, L63-L66, 2003.

Gopalswamy, N.: Coronal mass ejections of solar cycle 23, J. Astrophys. Astron., 27, 243-254, doi:10.1007/BF02702527, 2006.

Gosling, J. T.: Coronal mass ejections and magnetic flux ropes in interplanetary space, in: Physics of Magnetic Flux Ropes, Geophys. Monogr., 58, edited by: Priest, E. R., Lee, L. C., and Russell, C. T., 343-364, 1990.

Gosling, J. T., McComas, D. J., Phillips, J. L., and Bame, S. J.: Geomagnetic activity associated with earth passage of interplanetary shock disturbances and coronal mass ejections, J. Geophys. Res., 96, 7831-7839, 1991.

Hoeksema, J. T.: Large-scale solar and magnetic fields, Adv. Space Res., 11, 15-24, 1991.

$\mathrm{Hu}, \mathrm{Q}$. and Sonnerup, B. U. O.: Reconstruction of magnetic clouds in the solar wind: Orientations and configurations, J. Geophys. Res., 107(A7), 1142, doi:10.1029/2001JA000293, 2002.

Hundhausen, A. J.: Size and locations of coronal mass ejections: SMM observations from 1980 and 1984-1989, J. Geophys. Res., 98, 13177-13200, doi:10.1029/93JA00157, 1993.

Huttunen, K. E. J, Koskinen, H. E. J., and Schwenn, R.: Variability of magnetospheric storms driven by different solar wind perturbations, J. Geophys. Res., 107, 1121, doi:10.1029/2001JA900171, 2002.

Kaiser, M., Kucera, T. A., Davila, J. M., St. Cyr, O. C., Guhathakurta, M., and Christian, E: The STEREO mission: an introduction, Space Sci. Rev., 136, 1-4, doi:10.1007/s11214007-9277-0, 2007.

Kilpua, E. K. J., Luhmann, J. G., Gosling, J., Li, Y., Elliott, H., Russell, C. T., Jian, L., Galvin, A. B., Larson, D., Schroeder, P., Simunac, K., and Petrie, G.: Small Solar Wind Transients and Their Connection to the Large-Scale Coronal Structure, Sol. Phys., 256, 327-344, 2009a.

Kilpua, E. K. J., Pomoell, J., Vourlidas, A., Vainio, R., Luhmann, J., Li, Y., Schroeder, P., Galvin, A. B., and Simunac, K.: STEREO observations of interplanetary coronal mass ejections and prominence deflection during solar minimum period, Ann. Geophys., 27, 4491-4503, doi:10.5194/angeo-27-4491-2009, 2009b.

Lee, C. O., Luhmann, J. G., Zhao, X. P., Liu, Y., Riley, P., Arge, C. N., Russell, C. T., and de Pater, I.: Effects of the weak polar fields of solar cycle 23: investigation using OMNI for the STEREO mission period, Sol. Phys., 256: 345-363, doi:10.1007/s11207009-9345-6, 2009.

Lee, C. O., Luhmann, J. G., de Pater, I., Mason, G. M., Haggerty, D., Richardson, I. G., Cane, H. V., Jian, L. K., Russell, C. T., and Desai, M. I.: Organization of energetic particles by the solar wind structure during the declining to minimum phase of solar cycle 23, Sol. Phys, 263, 239-261, doi:10.1007/s11207-0109556-x, 2010.

Lee, C. O., Luhmann, J. G, Hoeksema, J. T., Sun, X., Arge, C. N., and de Pater, I.: Coronal field opens at lower height during the solar cycles 22 and 23 minimum periods: IMF comparison suggests source surface should be lowered, Sol. Phys., 269, 367388, doi:10.1007/s11207-010-9699-9, 2011.

Lepping, R. P., Berdichevsky, D. B., Wu, C.-C., Szabo, A., Narock, T., Mariani, F., Lazarus, A. J., and Quivers, A. J.: A summary of WIND magnetic clouds for years 1995-2003: model-fitted parameters, associated errors and classifications, Ann. Geophys., 24, 215-245, doi:10.5194/angeo-24-215-2006, 2006.

Luhmann, J. G., Lee, C. O., Li, Y., Arge, C. N., Galvin, A. B., Simunac, K., Russell, C. T., Howard, R. A., and Petrie, G.: Solar wind sources in the late declining phase of cycle 23: Effects of the weak solar polar field on high speed streams, Sol. Phys., 256, 285-305, doi:10.1007/s11207-009-9354-5, 2009.

McComas, D. J., Ebert, R. W., Elliott, H. A., Goldstein, B. E., Gosling, J. T., Schwadron, N. A., and Skoug, R. M.: Weaker solar wind from the polar coronal holes and the whole Sun, Geophys. Res. Lett., 35, L18103, doi:10.1029/2008GL034896, 2008.

Moldwin, M. B., Ford, S., Lepping R., Slavin, J., and Szabo, A.: Small-scale magnetic flux ropes in the solar wind, Geophys. Res. Lett., 27, 57-60, doi:10.1029/1999GL010724, 2000.

Neugebauer, M. and Goldstein, R.: Particle and field signatures of coronal mass ejections in the solar wind, in: Coronal Mass Ejections, Geophys. Monogr. 99, edited by: Crooker, N., Joselyn J. A., andFeynman, J., AGU, 245-251, 1997.

Owens, M. J., Schwadron, N. A., Crooker, N. U., Hughes, W. J., and Spence, H. E.: Role of coronal mass ejections in the heliospheric Hale cycle, Geophys. Res. Lett., 34, L06104, doi:10.1029/2006GL028795, 2007.

Plunkett, S. P., Thompson, B. J., St. Cyr, O. C., and Howard, R. A.: Solar source regions of coronal mass ejections and their geomagnetic effects, J. Geophys. Res., 63, 389-402, doi:10.1016/S13646826(00)00166-8, 2001.

Qiu, J., Hu, Q., Howard, T. A., and Yurchyshyn, V. B.: On the Magnetic flux budget in low-corona magnetic reconnection and interplanetary coronal mass ejections, Astrophys. J., 659, 758772, 2007.

Richardson, I. G. and Cane, H. V.: Regions of abnormally low proton temperature in the solar wind (1965-1991) and their association with ejecta, J. Geophys. Res., 100, 23397-23412, doi:10.1029/95JA02684, 1995.

Richardson, I. G. and Cane, H. V.: The fraction of interplanetary coronal mass ejections that are magnetic clouds: Evidence for a solar cycle variation, Geophys. Res. Lett., 31, L18804, 
doi:10.1029/2004GL020958, 2004.

Richardson, I. G. and Cane, H. V.: Near-Earth interplanetary coronal mass ejections during solar cycle 23 (1996-2009): catalog and summary of properties, Sol. Phys., 264, 189-237, doi:10.1007/s11207-010-9568-6, 2010.

Riley, P., Schatzman, C., Cane, H. V., Richardson, I. G., and Gopalswamy, N.: On the rates of coronal mass ejections: Remote solar and in situ observations, Astrophys. J., 647, 648-653, 2006.

Rouillard, A. P., Savani, N., Davies, J. A., Lavraud, B., Forsyth, R. J., Morley, S. K., Opitz, A., Sheeley, N. R., Sauvaud, J.-A., Simunac, K. D. C., Luhmann, J. G., Galvin, A. G., Crothers, S. R., Davis, S. J., Harrison, R. A., Lockwood, M., Eyles, C. J., Bewsher, D., and Brown, D. S.: A Multispacecraft analysis of small scale transient entrained by solar wind streams, Sol. Phys., 256, 307-326, 2009.

Sanderson, T. R., Lin, R. P., Larson, D., McCarthy, M. P., Parks, G. K., Bosqued, J. M., Lormant, N., Ogilvie, K., Lepping, R. P., Szabo, A., Lazarus, A. J., Steinberg, J., and Hoeksema, J. T.: Wind observations of the influence of the Sun's magnetic field on the interplanetary medium at $1 \mathrm{AU}, \mathrm{J}$. Geophys. Res., 103, 17235-17248, doi:10.1029/97JA02884, 1998.

Schatten, K. H., Wilcox, J. M., and Ness, N. F.: A model of interplanetary and coronal magnetic fields, Sol. Phys., 6, 442-455, 1969.

Sheeley Jr., N. R., Herbst, A. D., Palatchi, C. A., Wang, Y.-M., Howard, R. A., Moses, J. D., Vourlidas, A., Newmark, J. S., Socker, D. G., Plunkett, S. P., Korendyke, C. M., Burlaga, L. F., Davila, J. M., Thompson, W. T., St Cyr, O. C., Harrison, R. A., Davis, C. J., Eyles, C. J., Halain, J. P., Wang, D., Rich, N. B., Battams, K., Esfandiari, E., and Stenborg, G.: Heliospheric images of the solar wind at Earth, Astrophys. J., 675, 853-862, 2008.

Smith, E. J. and Balogh, A.: Decrease in heliospheric magnetic flux in this solar minimum: recent Ulysses magnetic field observations, Geophys. Res. Lett. 35, L22103, doi:10.1029/2008GL035345, 2008.
St. Cyr, O. C., Howard, R. A., Sheeley, N. R., Plunkett, S. P., Michels, D. J., Paswaters, S. E., Koomen, M. J., Simnett, G. M., Thomson, B. J., Gurman, J. B., Schwenn, R., Webb, D. F., Hildner, E., and Lamy, P. L.: Properties of coronal mass ejections: SOHO LASCO observations from January 1996 to June 1998, J. Geophys. Res., 105, 18169-18185, doi:10.1029/1999JA000381, 2000.

Vourlidas, A., Howard, R. A., Esfandiari, E., Patsourakos, S., Yashiro, S., and Michalek, G.: Comprehensive analysis of coronal mass ejection mass and energy properties over a full solar cycle, Astrophys. J., 722, 1522-1538, 2010.

Vourlidas, A., Howard, R. A., Esfandiari, E., Patsourakos, S., Yashiro, S., and Michalek, G.: Erratum: "Comprehensive analysis of coronal mass ejection mass and energy properties over a full solar cycle", Astrophys. J., 730, 59-61, 2011.

Wang, Y.-M., Sheeley Jr., N., and Rich, N. B.: Coronal pseudostreamerse, Astrophys. J., 658, 1340-1348, 2007.

Wang, Y.-M., Robbrecht, E., and Sheeley Jr., N. R.: On the weakening of the polar magnetic fields during solar cycle 23, Astrophys. J., 707, 1372-1386, 2009.

Xie, H., St. Cy, O. C., Gopalswamy, N., Yashiro, S., Krall, J., Kramar, M., and Davila, J.: On the origin, 3D structure and dynamic evolution of CMEs near solar minimum, Sol. Phys., 259, 143161, doi:10.1007/s11207-009-9422-x, 2009.

Yashiro, S., Michalek, G., and Gopalswamy, N.: A comparison of coronal mass ejections identified by manual and automatic methods, Ann. Geophys., 26, 3103-3112, doi:10.5194/angeo26-3103-2008, 2008.

Zhao, X. P. and Webb, D. F.: Source regions and storm effectiveness of frontside full halo, J. Geophys. Res., 108, 1234, doi:10.1029/2002JA009606, 2003.

Zurbuchen, T. H. and Richardson, I. G.: In-situ solar wind and magnetic field signatures of interplanetary coronal mass ejections, Space Sci. Rev., 123, 1572, doi:10.1007/s11214-006-9010-4, 2006. 